\title{
Accessing flavor-singlet quark and gluon parton distributions from lattice QCD
}

\author{
Jianhui Zhang* \\ Center of Advanced Quantum Studies, Department of Physics, Beijing Normal University, \\ Beijing 100875, China \\ E-mail: zhangjianhui@bnu.edu.cn
}

\begin{abstract}
Recent years have witnessed rapid progress on computing parton distribution functions (PDFs) from lattice QCD. Such computations have been focused on the isovector quark PDFs which do not involve mixing with gluon PDFs and therefore are the easiest to calculate. In this talk, I present recent developments that allow us to access flavor-singlet quark PDFs as well as gluon PDFs from lattice QCD.
\end{abstract}

37th International Symposium on Lattice Field Theory - Lattice2019

16-22 June 2019

Wuhan, China

${ }^{*}$ Speaker. 


\section{Introduction}

Parton distribution functions (PDFs) characterize the hadron structure at high-energy and are crucial inputs for making theoretical predictions of experimental observables at e.g. the LHC. They are defined as the expectation value of lightcone correlations in a hadron state and thus can not be readily computed on a Euclidean lattice. Currently, the most widely used approach to determine them is to assume a smoothly parametrized form and fit the unknown parameters to a large variety of experimental data (for a recent review, see e.g. Ref. [1]). Lattice efforts on determining them have been mainly focused on the computation of their moments, which are matrix elements of local operators. The PDFs can be reconstructed in principle if all their moments are known. However, to date only the first few moments can be calculated in lattice QCD $[2,3,4,5]$ due to power divergent mixing between different moments operators and increasing stochastic noise for high moments operators.

In the past few years, a new framework has been developed to circumvent the above difficulty, which is now known as large momentum effective theory (LaMET) [6, 7]. According to LaMET, the PDFs (as well as other parton observables), instead of its moments, can be accessed from lattice QCD using the following procedure: 1) Construct an appropriate static-operator matrix element (quasi-PDF) that approaches the PDF in the infinite momentum limit of the external hadron. The quasi-PDF constructed in this way is usually hadron-momentum-dependent but time-independent, and thus can be readily computed on the lattice. 2) Calculate the quasi-PDF on the lattice and renormalize it nonperturbatively in an appropriate scheme. 3) Match the renormalized quasi-PDF to the PDF through a factorization formula accurate up to power corrections that are suppressed by the hadron momentum [6, 7].

Since LaMET was proposed, much progress has been achieved both in the theoretical understanding of the formalism and in the direct calculation of PDFs from lattice QCD (see Ref. [8] and references therein). In particular, multiplicative renormalization of both the quark $[9,10,11]$ and the gluon $[12,13]$ quasi-PDF has been established in coordinate space. Nonperturbative renormalization in the regularization-independent momentum subtraction (RI/MOM) scheme as well as a perturbative matching in the same scheme has been carried out for the nonsinglet quark quasiPDFs $[14,15,16,17]$ (see also $[18,19,20]$ ), as well as for the singlet quark and gluon quasiPDFs [12, 21]. Despite limited volumes and relatively coarse lattice spacings, the state-of-the-art nucleon isovector quark PDFs determined from lattice data at the physical point have shown a reasonable agreement $[16,17,20]$ with phenomenological results extracted from the experimental data $[22,23,24,25,26]$. Of course, a careful study of theoretical uncertainties and lattice artifacts is still needed to fully establish the reliability of the results.

In this talk, I report recent theoretical progress in LaMET that enables us to extract both the flavor-singlet quark PDF and the gluon PDF from lattice QCD.

\section{Quasi-PDFs in LaMET}

In high-energy collisions, the PDFs are defined as the hadron matrix elements of quark and gluon nonlocal correlators along the lightcone. For example, the unpolarized quark distribution is 
defined as

$$
f_{q_{i} / H}(x, \mu)=\int \frac{d \xi^{-}}{4 \pi} e^{-i x P^{+} \xi^{-}}\left\langle P\left|\bar{q}_{i}\left(\xi^{-}\right) \gamma^{+} W\left(\xi^{-}, 0\right) q_{i}(0)\right| P\right\rangle
$$

for a given flavor $i$, where $x=k^{+} / P^{+}$is the longitudinal momentum fraction carried by the quark of flavor $i$. $\mu$ is the renormalization scale in the $\overline{\mathrm{MS}}$ scheme, $P^{\mu}=\left(P^{0}, 0,0, P^{z}\right)$ is the hadron momentum, $\xi^{ \pm}=(t \pm z) / \sqrt{2}$ are the lightcone coordinates, and $W\left(\xi^{-}, 0\right)=\exp \left(-i g \int_{0}^{\xi^{-}} d \eta^{-} A^{+}\left(\eta^{-}\right)\right)$ is the Wilson line inserted to maintain the gauge invariance of the nonlocal correlator. $A^{+}=A_{a}^{+} t^{a}$ with $t^{a}$ being the generators in the fundamental representation of color $S U$ (3) group.

Analogously, the unpolarized gluon distribution can be defined as [27]

$$
f_{g / H}(x, \mu)=\int \frac{d \xi^{-}}{2 \pi x P^{+}} e^{-i x P^{+} \xi^{-}}\left\langle P\left|F_{a}^{+i}\left(\xi^{-}\right) \mathscr{W}\left(\xi^{-}, 0\right) F_{a}^{+i}(0)\right| P\right\rangle,
$$

where $F_{a}^{\mu v}=\partial^{\mu} A_{a}^{v}-\partial^{v} A_{a}^{\mu}-g f_{a b c} A_{b}^{\mu} A_{c}^{v}$ is the gluon field strength, and $i$ runs over the transverse indices. The above Wilson line $\mathscr{W}$ takes a similar form as the quark case, but is defined in the adjoint representation.

The above quark and gluon PDFs can not be directly computed on the lattice due to their real-time dependence. However, according to LaMET, they can be extracted from lattice calculations of appropriately constructed quasi-PDFs via a factorization or matching procedure. For the unpolarized quark PDF, a well-suited quasi-PDF candidate is given by

$$
\tilde{f}_{q_{i} / H}\left(x, \mu, P^{z}\right)=N \int \frac{d z}{4 \pi} e^{i z x P^{z}}\left\langle P\left|\bar{q}_{i}(z) \Gamma W(z, 0) q_{i}(0)\right| P\right\rangle,
$$

where $z$ is a spatial direction, $\Gamma=\left\{\gamma^{z}, \gamma^{t}\right\}$ is a Dirac matrix with the corresponding normalization factor $N=\left\{1, P^{z} / P^{t}\right\}$, respectively. The choice with $\Gamma=\gamma^{t}$ has the advantage of avoiding mixing with the scalar PDF when a non-chiral lattice fermion is used [18, 28].

For the unpolarized gluon PDF, we have also identified four operators [12] that are multiplicatively renormalizable and thus are suitable to define the corresponding quasi-PDFs. These operators are

$$
\begin{array}{ll}
O_{g}^{(1)}(z, 0) \equiv F^{t i}(z) \mathscr{W}(z, 0) F_{i}^{t}(0), & O_{g}^{(2)}(z, 0) \equiv F^{z i}(z) \mathscr{W}(z, 0) F_{i}{ }^{z}(0), \\
O_{g}^{(3)}(z, 0) \equiv F^{t i}(z) \mathscr{W}(z, 0) F_{i}{ }^{z}(0), & O_{g}^{(4)}(z, 0) \equiv F^{z \mu}(z) \mathscr{W}(z, 0) F_{\mu}^{z}(0),
\end{array}
$$

where a summation over transverse (all) components is implied for $i(\mu)$. The corresponding gluon quasi-PDF is then defined as

$$
\tilde{f}_{g / H}^{(n)}\left(x, \mu, P^{z}\right)=N^{(n)} \int \frac{d z}{2 \pi x P^{z}} e^{i z x P^{z}}\left\langle P\left|O_{g}^{(n)}(z, 0)\right| P\right\rangle,
$$

with the normalization factors being given by

$$
N^{(2)}=N^{(4)}=1, \quad N^{(1)}=\frac{\left(P^{z}\right)^{2}}{\left(P^{t}\right)^{2}}, \quad N^{(3)}=\frac{P^{z}}{P^{t}} .
$$

All above gluon quasi-PDF operators are defined in terms of an adjoint gauge link. Alternatively, they can be parametrized using gauge links in the fundamental representation $U\left(z_{2}, z_{1}\right)$ [29]. Taking the operator $O_{g}^{(3)}$ as an example, one could use

$$
O_{g}^{(3)}\left(z_{2}, z_{1}\right)=2 \operatorname{Tr}\left[F^{t i}\left(z_{2}\right) U\left(z_{2}, z_{1}\right) F_{i}^{z}\left(z_{1}\right) U\left(z_{1}, z_{2}\right)\right] .
$$


Here $F^{\mu v}=F_{\mu v}^{a} t^{a}$ and $t^{a}$ is the generator in the fundamental representation with $\operatorname{tr}\left[t^{a} t^{b}\right]=1 / 2 \delta^{a b}$. Eq. (2.7) makes the implementation on the lattice simpler. The results presented below are mainly focused on the definition in Eq. (2.4), but the results also apply to Eq. (2.7).

\section{Renormalization and Factorization}

The nonlocal quasi-PDF operators at different $z$ do not mix under renormalization due to their multiplicative renormalizability. This allows us to carry out a nonperturbative renormalization of the quasi-PDF by calculating the renormalization factors as a whole for each $z$. This is analogous to the renormalization of local composite operators, which is usually carried out in the RI/MOM scheme [30] on the lattice. In the RI/MOM scheme, the renormalization of local composite operators is done by demanding that the counterterm cancels all loop contributions to their matrix element between off-shell external states at specific momenta $[14,15]$. For multiplicatively renormalizable nonlocal quasi-PDF operators, the renormalization is similar but now one requires calculating the renormalization factors at each $z$.

In principle, the quark and gluon quasi-PDFs can be renormalized separately and then matched to the PDFs. However, in Ref. [12] we found that taking into account the finite mixing between them at the renormalization stage will help improve the convergence in the implementation of the matching in the RI/MOM scheme. To this end, it suffices to consider the following mixing of quasi-PDFs

$$
\left(\begin{array}{c}
O_{g}^{(n)}(z, 0) \\
O_{q}^{s}(z, 0)
\end{array}\right)=\left(\begin{array}{cc}
Z_{11}(z) & Z_{12}(z) / z \\
z Z_{21}(z) & Z_{22}(z)
\end{array}\right)\left(\begin{array}{c}
O_{g, R}^{(n)}(z, 0) \\
O_{q, R}^{s}(z, 0)
\end{array}\right),
$$

where $O_{q}^{s}\left(z_{1}, z_{2}\right)=1 / 2\left[\bar{q}_{i}\left(z_{1}\right) \Gamma W\left(z_{1}, z_{2}\right) q_{i}\left(z_{2}\right)-\left(z_{1} \leftrightarrow z_{2}\right)\right]$ is the $C$-even combination of quark operators, $Z_{i j}(z)$ are dimensionless factors, and $z$ compensates for the different mass dimension between the quark and gluon quasi-PDF operators. In the limit $z \rightarrow 0$ (taken after combining the entries of the mixing matrix and the operators), the above mixing pattern reduces to the mixing pattern of local operators.

The renormalization factors in the above mixing matrix can be determined using the following renormalization conditions

$$
\begin{aligned}
& \left.\frac{\operatorname{Tr}\left[\Lambda_{22}(p, z) \mathscr{P}\right]_{R}}{\operatorname{Tr}\left[\Lambda_{22}(p, z) \mathscr{P}\right]_{\text {tree }}}\right|_{\substack{p^{2}=-\mu_{R}^{2} \\
p_{z}=p_{z}^{p}}}=1,\left.\quad \frac{\left[P_{i j}^{a b} \Lambda_{11}^{a b, i j}(p, z)\right]_{R}}{\left[P_{i j}^{a b} \Lambda_{11}^{a b, i j}(p, z)\right]_{\text {tree }}}\right|_{\begin{array}{c}
p^{2}=-\mu_{R}^{2} \\
p_{z}=p_{z}^{R}
\end{array}}=1, \\
& \left.\operatorname{Tr}\left[\Lambda_{12}(p, z) \mathscr{P}\right]_{R}\right|_{\substack{p^{2}=-\mu_{R}^{2} \\
p_{z}=p_{z}^{R}}}=0,\left.\quad\left[P_{i j}^{a b} \Lambda_{21}^{a b, i j}(p, z)\right]_{R}\right|_{\begin{array}{c}
p^{2}=-\mu_{R}^{2} \\
p_{z}=p_{z}^{R}
\end{array}}=0,
\end{aligned}
$$

where $\Lambda_{\{11,12\}}\left(\Lambda_{\{21,22\}}\right)$ denote the amputated Green's functions of $O_{g}^{(n)}\left(O_{q}^{s}\right)$ in an offshell gluon and quark state, respectively. $\mathscr{P}$ and $P_{i j}^{a b}$ are projection operators that are associated with the quark and gluon matrix elements and define the RI/MOM renormalization factors. $\mu_{R}$ and $p_{z}^{R}$ are unphysical scales introduced in the RI/MOM scheme to specify the subtraction point. $b, c$ are color indices and $i, j$ Lorentz indices. In the nonsinglet quark PDF case with $\Gamma=\gamma^{t}$ [31], the amputated Green's function has the following structure

$$
\Lambda_{\gamma^{t}}(p, z)=\widetilde{f}_{t}(p, z) \gamma^{t}+\widetilde{f}_{z}(p, z) \frac{p^{t} \gamma^{z}}{p^{z}}+\widetilde{f}_{p}(p, z) \frac{p^{t} \not p}{p^{2}}
$$


and $\mathscr{P}$ was chosen there in such a way that it projects out the coefficient of $\gamma^{t}$ only, which captures all terms in $\Lambda_{\gamma^{t}}(p, z)$ that lead to UV divergences in the local limit. However, in general both the coefficient of $\gamma^{t}$ and $\gamma^{z}$ can lead to UV divergences in the local limit. This is the case e.g. in the mixing diagrams. Thus, in the present case we will need to project out both coefficients to define the RI/MOM counterterm. As for $P_{i j}^{a b}$, a simple choice is $P_{i j}^{a b}=\delta^{a b} g_{\perp, i j} /(2-D)$, where $g_{\perp, i j}$ denotes the transverse metric tensor and $D$ is the spacetime dimension.

Defining the inverse of the renormalization matrix in Eq. (3.1) as

$$
\overline{\mathscr{Z}}=\left(\begin{array}{cc}
\bar{Z}_{11}(z) & \bar{Z}_{12}(z) / z \\
z \bar{Z}_{21}(z) & \bar{Z}_{22}(z)
\end{array}\right)=\left(\begin{array}{cc}
Z_{11}(z) & Z_{12}(z) / z \\
z Z_{21}(z) & Z_{22}(z)
\end{array}\right)^{-1}
$$

we then have from Eqs. (3.1), (3.2) and (3.4)

$$
\begin{aligned}
& \bar{Z}_{11}(z)=\left.\frac{\left[P_{i j}^{a b} \Lambda_{11}^{a b, i j}(p, z)\right]_{\text {tree }} \operatorname{Tr}\left[\Lambda_{22}(p, z) \mathscr{P}\right]}{\left(\left[P_{i j}^{a b} \Lambda_{11}^{a b, i j}(p, z)\right] \operatorname{Tr}\left[\Lambda_{22}(p, z) \mathscr{P}\right]-\left[P_{i j}^{a b} \Lambda_{21}^{a b, i j}(p, z)\right] \operatorname{Tr}\left[\Lambda_{12}(p, z) \mathscr{P}\right]\right)}\right|_{\substack{p^{2}=-\mu_{R}^{2} \\
p_{z}=p_{z}^{R}}}, \\
& \bar{Z}_{12}(z) / z=-\left.\frac{\left[P_{i j}^{a b} \Lambda_{11}^{a b, i j}(p, z)\right]_{\operatorname{tree}} \operatorname{Tr}\left[\Lambda_{12}(p, z) \mathscr{P}\right]}{\left(\left[P_{i j}^{a b} \Lambda_{11}^{a b, i j}(p, z)\right] \operatorname{Tr}\left[\Lambda_{22}(p, z) \mathscr{P}\right]-\left[P_{i j}^{a b} \Lambda_{21}^{a b, i j}(p, z)\right] \operatorname{Tr}\left[\Lambda_{12}(p, z) \mathscr{P}\right]\right)}\right|_{\substack{p^{2}=-\mu_{R}^{2} \\
p_{z}=p_{z}^{R}}}, \\
& z \bar{Z}_{21}(z)=-\left.\frac{\left[P_{i j}^{a b} \Lambda_{21}^{a b, i j}(p, z)\right] \operatorname{Tr}\left[\Lambda_{22}(p, z) \mathscr{P}\right]_{\text {tree }}}{\left(\left[P_{i j}^{a b} \Lambda_{11}^{a b, i j}(p, z)\right] \operatorname{Tr}\left[\Lambda_{22}(p, z) \mathscr{P}\right]-\left[P_{i j}^{a b} \Lambda_{21}^{a b, i j}(p, z)\right] \operatorname{Tr}\left[\Lambda_{12}(p, z) \mathscr{P}\right]\right)}\right|_{\substack{p^{2}=-\mu_{R}^{2} \\
p_{z}=p_{z}^{R}}}, \\
& \bar{Z}_{22}(z)=\left.\frac{\left[P_{i j}^{a b} \Lambda_{11}^{a b, i j}(p, z)\right] \operatorname{Tr}\left[\Lambda_{22}(p, z) \mathscr{P}\right]_{\text {tree }}}{\left(\left[P_{i j}^{a b} \Lambda_{11}^{a b, i j}(p, z)\right] \operatorname{Tr}\left[\Lambda_{22}(p, z) \mathscr{P}\right]-\left[P_{i j}^{a b} \Lambda_{21}^{a b, i j}(p, z)\right] \operatorname{Tr}\left[\Lambda_{12}(p, z) \mathscr{P}\right]\right)}\right|_{\substack{p^{2}=-\mu_{R}^{2} \\
p_{z}=p_{z}^{R}}} .
\end{aligned}
$$

Denoting the hadron matrix element of $O(z, 0)$ as $h\left(z, P^{z}, 1 / a\right)$, i.e., $h_{i}\left(z, P^{z}, 1 / a\right)=\left\langle P\left|O_{i}(z, 0)\right| P\right\rangle$, $i=q, g$, the renormalized hadron matrix elements then read

$$
\begin{aligned}
& h_{g, R}^{(n)}\left(z, P^{z}, \mu_{R}, p_{z}^{R}\right)=\bar{Z}_{11}\left(z, \mu_{R}, p_{z}^{R}, 1 / a\right) h_{g}^{(n)}\left(z, P^{z}, 1 / a\right)+\bar{Z}_{12}\left(z, \mu_{R}, p_{z}^{R}, 1 / a\right) / z h_{q}^{s}\left(z, P^{z}, 1 / a\right), \\
& h_{q, R}^{s}\left(z, P^{z}, \mu_{R}, p_{z}^{R}\right)=\bar{Z}_{22}\left(z, \mu_{R}, p_{z}^{R}, 1 / a\right) h_{q}^{s}\left(z, P^{z}, 1 / a\right)+z \bar{Z}_{21}\left(z, \mu_{R}, p_{z}^{R}, 1 / a\right) h_{g}^{(n)}\left(z, P^{z}, 1 / a\right) .(3.6)
\end{aligned}
$$

The renormalized quasi-PDF in the RI/MOM scheme can be obtained from the above renormalized matrix elements by a Fourier transform given in Eqs. (2.3) and (2.5), respectively. Note that we can take the continuum limit $a \rightarrow 0$ in $h_{R}$ since all terms singular in $a$ have been removed by the renormalization procedure. This means that the factorization of the renormalized matrix element can be studied in the continuum.

In Ref. [21], we have derived a general factorization formula for the renormalized matrix element above as well as for their Fourier transform, the quark and gluon quasi-PDFs, in the presence 
of mixing. As an example, we show the factorization of the latter

$$
\begin{aligned}
\tilde{f}_{g / H}^{(n)}\left(x, P^{z}, p_{z}^{R}, \mu_{R}\right)= & \int_{-1}^{1} \frac{d y}{|y|}\left[C_{g g}\left(\frac{x}{y}, \frac{\mu_{R}}{p_{z}^{R}}, \frac{y P^{z}}{\mu}, \frac{y P^{z}}{p_{z}^{R}}\right) f_{g / H}(y, \mu)+C_{g q}\left(\frac{x}{y}, \frac{\mu_{R}}{p_{z}^{R}}, \frac{y P^{z}}{\mu}, \frac{y P^{z}}{p_{z}^{R}}\right) f_{q_{j} / H}(y, \mu)\right] \\
& +\mathscr{O}\left(\frac{M^{2}}{P_{z}^{2}}, \frac{\Lambda_{\mathrm{QCD}}^{2}}{x^{2} P_{z}^{2}}\right), \\
\tilde{f}_{q_{i} / H}\left(x, P^{z}, p_{z}^{R}, \mu_{R}\right)= & \int_{-1}^{1} \frac{d y}{|y|}\left[C_{q_{i} q_{j}}\left(\frac{x}{y}, \frac{\mu_{R}}{p_{z}^{R}}, \frac{y P^{z}}{\mu}, \frac{y P^{z}}{p_{z}^{R}}\right) f_{q_{j} / H}(y, \mu)+C_{q g}\left(\frac{x}{y}, \frac{\mu_{R}}{p_{z}^{R}}, \frac{y P^{z}}{\mu}, \frac{y P^{z}}{p_{z}^{R}}\right) f_{g / H}(y, \mu)\right] \\
& +\mathscr{O}\left(\frac{M^{2}}{P_{z}^{2}}, \frac{\Lambda_{\mathrm{QCD}}^{2}}{x^{2} P_{z}^{2}}\right),
\end{aligned}
$$

where a summation of $j$ over all quark flavors is implied. The matching coefficients $C_{i j}$ have also been computed to one-loop in Ref. [21]. With the above formulas, we are in principle able to extract the quark and gluon PDFs on the r.h.s. from the quasi-PDFs on the 1.h.s.

\section{Conclusion}

To conclude, we have presented recent theoretical studies on the renormalization and factorization of quark and gluon quasi-PDFs in the presence of mixing. This in principle allows us to extract flavor-singlet quark PDFs as well as gluon PDFs from lattice calculations of the corresponding quasi-PDFs.

\section{References}

[1] J. Gao, L. Harland-Lang and J. Rojo, Phys. Rept. 742, 1 (2018) doi:10.1016/j.physrep.2018.03.002 [arXiv:1709.04922 [hep-ph]].

[2] G. Martinelli and C. T. Sachrajda, Phys. Lett. B 196, 184 (1987). doi:10.1016/0370-2693(87)90601-0

[3] G. Martinelli and C. T. Sachrajda, Phys. Lett. B 217, 319 (1989). doi:10.1016/0370-2693(89)90874-5

[4] W. Detmold, W. Melnitchouk and A. W. Thomas, Eur. Phys. J. direct 3, no. 1, 13 (2001) doi:10.1007/s1010501c0013 [hep-lat/0108002].

[5] D. Dolgov et al. [LHPC and TXL Collaborations], Phys. Rev. D 66, 034506 (2002) doi:10.1103/PhysRevD.66.034506 [hep-lat/0201021].

[6] X. Ji, Phys. Rev. Lett. 110, 262002 (2013) doi:10.1103/PhysRevLett.110.262002 [arXiv:1305.1539 [hep-ph]].

[7] X. Ji, Sci. China Phys. Mech. Astron. 57, 1407 (2014) doi:10.1007/s11433-014-5492-3 [arXiv:1404.6680 [hep-ph]].

[8] K. Cichy and M. Constantinou, Adv. High Energy Phys. 2019, 3036904 (2019) doi:10.1155/2019/3036904 [arXiv:1811.07248 [hep-lat]].

[9] X. Ji, J. H. Zhang and Y. Zhao, Phys. Rev. Lett. 120, no. 11, 112001 (2018) doi:10.1103/PhysRevLett.120.112001 [arXiv:1706.08962 [hep-ph]].

[10] T. Ishikawa, Y. Q. Ma, J. W. Qiu and S. Yoshida, Phys. Rev. D 96, no. 9, 094019 (2017) doi:10.1103/PhysRevD.96.094019 [arXiv:1707.03107 [hep-ph]]. 
[11] J. Green, K. Jansen and F. Steffens, Phys. Rev. Lett. 121, no. 2, 022004 (2018) doi:10.1103/PhysRevLett.121.022004 [arXiv:1707.07152 [hep-lat]].

[12] J. H. Zhang, X. Ji, A. Schäfer, W. Wang and S. Zhao, arXiv:1808.10824 [hep-ph].

[13] Z. Y. Li, Y. Q. Ma and J. W. Qiu, Phys. Rev. Lett. 122, no. 6, 062002 (2019) doi:10.1103/PhysRevLett.122.062002 [arXiv:1809.01836 [hep-ph]].

[14] J. W. Chen, T. Ishikawa, L. Jin, H. W. Lin, Y. B. Yang, J. H. Zhang and Y. Zhao, Phys. Rev. D 97, no. 1, 014505 (2018) doi:10.1103/PhysRevD.97.014505 [arXiv:1706.01295 [hep-lat]].

[15] I. W. Stewart and Y. Zhao, Phys. Rev. D 97, no. 5, 054512 (2018) doi:10.1103/PhysRevD.97.054512 [arXiv:1709.04933 [hep-ph]].

[16] J. W. Chen, L. Jin, H. W. Lin, Y. S. Liu, Y. B. Yang, J. H. Zhang and Y. Zhao, arXiv:1803.04393 [hep-lat].

[17] H. W. Lin et al., Phys. Rev. Lett. 121, no. 24, 242003 (2018) doi:10.1103/PhysRevLett.121.242003 [arXiv:1807.07431 [hep-lat]].

[18] M. Constantinou and H. Panagopoulos, Phys. Rev. D 96, no. 5, 054506 (2017) doi:10.1103/PhysRevD.96.054506 [arXiv:1705.11193 [hep-lat]].

[19] C. Alexandrou, K. Cichy, M. Constantinou, K. Hadjiyiannakou, K. Jansen, H. Panagopoulos and F. Steffens, Nucl. Phys. B 923, 394 (2017) doi:10.1016/j.nuclphysb.2017.08.012 [arXiv:1706.00265 [hep-lat]].

[20] C. Alexandrou, K. Cichy, M. Constantinou, K. Jansen, A. Scapellato and F. Steffens, Phys. Rev. Lett. 121, no. 11, 112001 (2018) doi:10.1103/PhysRevLett.121.112001 [arXiv:1803.02685 [hep-lat]].

[21] W. Wang, J. H. Zhang, S. Zhao and R. Zhu, Phys. Rev. D 100, no. 7, 074509 (2019) doi:10.1103/PhysRevD.100.074509 [arXiv:1904.00978 [hep-ph]].

[22] S. Dulat et al., Phys. Rev. D 93, no. 3, 033006 (2016) doi:10.1103/PhysRevD.93.033006 [arXiv:1506.07443 [hep-ph]].

[23] R. D. Ball et al. [NNPDF Collaboration], Eur. Phys. J. C 77, no. 10, 663 (2017) doi:10.1140/epjc/s10052-017-5199-5 [arXiv:1706.00428 [hep-ph]].

[24] L. A. Harland-Lang, A. D. Martin, P. Motylinski and R. S. Thorne, Eur. Phys. J. C 75, no. 5, 204 (2015) doi:10.1140/epjc/s10052-015-3397-6 [arXiv:1412.3989 [hep-ph]].

[25] E. R. Nocera et al. [NNPDF Collaboration], Nucl. Phys. B 887, 276 (2014) doi:10.1016/j.nuclphysb.2014.08.008 [arXiv:1406.5539 [hep-ph]].

[26] J. J. Ethier, N. Sato and W. Melnitchouk, Phys. Rev. Lett. 119, no. 13, 132001 (2017) doi:10.1103/PhysRevLett.119.132001 [arXiv:1705.05889 [hep-ph]].

[27] J. Collins, Camb. Monogr. Part. Phys. Nucl. Phys. Cosmol. 32, 1 (2011).

[28] J. W. Chen, T. Ishikawa, L. Jin, H. W. Lin, Y. B. Yang, J. H. Zhang and Y. Zhao, arXiv:1710.01089 [hep-lat].

[29] H. Dorn, Fortsch. Phys. 34, 11 (1986). doi:10.1002/prop.19860340104

[30] G. Martinelli, C. Pittori, C. T. Sachrajda, M. Testa and A. Vladikas, Nucl. Phys. B 445, 81 (1995) doi:10.1016/0550-3213(95)00126-D [hep-lat/9411010].

[31] Y. S. Liu, J. W. Chen, L. Jin, H. W. Lin, Y. B. Yang, J. H. Zhang and Y. Zhao, arXiv:1807.06566 [hep-lat]. 